\section{Dlk1 Promotes a Fast Motor Neuron Biophysical Signature Required for Peak Force Execution}

\author{
Daniel Müller, ${ }^{1}$ Pitchaiah Cherukuri, ${ }^{1}$ Kristine Henningfeld, ${ }^{2,3}$ Chor Hoon Poh, ${ }^{1}$ Lars Wittler, ${ }^{4}$ \\ Phillip Grote, ${ }^{4}$ Oliver Schlüter, ${ }^{5}$ Jennifer Schmidt, ${ }^{6}$ jorge Laborda, ${ }^{7}$ Steven R. Bauer, ${ }^{8}$ \\ Robert M. Brownstone, ${ }^{9}$ Till Marquardt ${ }^{1,3}$ *
}

Motor neurons, which relay neural commands to drive skeletal muscle movements, encompass types ranging from "slow" to "fast," whose biophysical properties govern the timing, gradation, and amplitude of muscle force. Here we identify the noncanonical Notch ligand Delta-like homolog 1 (Dlk1) as a determinant of motor neuron functional diversification. Dlk1, expressed by $\sim 30 \%$ of motor neurons, is necessary and sufficient to promote a fast biophysical signature in the mouse and chick. Dlk1 suppresses Notch signaling and activates expression of the $\mathrm{K}^{+}$channel subunit Keng4 to modulate delayed-rectifier currents. Dlk1 inactivation comprehensively shifts motor neurons toward slow biophysical and transcriptome signatures, while abolishing peak force outputs. Our findings provide insights into the development of motor neuron functional diversity and its contribution to the execution of movements.

$\mathrm{S}$ low or fast motor neurons respectively synapse with type I muscle fibers responsible for fatigue-resistant low-force contractions or fatigable type IIb muscle fibers eliciting brief high-force outputs (fig. S1A) (1-3). The biophysical properties of these motor neuron types are exquisitely matched to the muscle fiber contractile properties $(1,4-9)$. For instance, slow motor neurons, which possess low activation thresholds and long afterhyperpolarizations, can sustain long periods of low-frequency firing $(1,4-9)$. Fast motor neurons, in contrast, are larger, exhibit high activation thresholds with shorter afterhyperpolarizations, and can fire in high-frequency bursts (1, 4-9). Motor neurons with properties falling between these two extremes (which we call intermediate motor neurons) innervate muscle fibers with similarly intermediate characteristics $(3-6,9)$. We identified molecular markers for these motor neuron types and studied how motor neuron functional diversity is established.

We exploited the distinct fiber type composition of soleus, tibialis anterior, and quadriceps muscles in the early postnatal mouse hindlimb (fig.

\footnotetext{
${ }^{1}$ Developmental Neurobiology Laboratory, European Neuroscience Institute (ENI-G), Grisebachstraße 5, 37077 Göttingen, Germany. ${ }^{2}$ Institute of Developmental Biochemistry, University Medical Center Göttingen, Justus-von-Liebig-Weg 11, 37077 Göttingen, Germany. ${ }^{3}$ Center for Nanoscale Microscopy and Molecular Physiology of the Brain, Göttingen, Germany. ${ }^{4}$ Department of Developmental Genetics, Max-Planck Institute for Molecular Genetics, Ihnestraße 63-73, 14195 Berlin, Germany. ${ }^{5}$ Molecular Neurobiology Laboratory, ENI-G, Grisebachstraße 5, 37077 Göttingen, Germany. ${ }^{6}$ Department of Biological Sciences, University of Illinois at Chicago, 900 South Ashland Avenue, MBRB 4210 Chicago, IL 60607, USA ${ }^{7}$ Departament of Inorganic and Organic Chemistry and Biochemistry, University of Castilla-La Mancha Medical School, 02006 C/Almansa 14, Albacete, Spain. ${ }^{8}$ Cellular and Tissue Therapies Branch, Division of Cellular and Gene Therapies, Center for Biologics and Research, U.S. Food and Drug Administration, Bethesda, MD 20892, USA ${ }^{9}$ Medical Staff, QEIl Health Sciences Centre, Departments of Surgery and Medical Neuroscience, Dalhousie University, Halifax B3H 4R2, Canada.

*Corresponding author. E-mail: t.marquardt@eni-g.de
}

S1B) to retrogradely label, isolate, and obtain transcriptome profiles of motor pools enriched in motor neurons developing into either slow/ intermediate or fast types (fig. S1, C to O). One of the genes associated with a fast motor pool profile encoded Dlk1 (fig. S1P), a type I transmembrane protein related to the Notch ligand Delta, which functions in adipogenesis, postnatal myogenesis, and adult neurogenesis (10-12). Dlk1 was selectively expressed by large $\alpha$ motor neurons, but not smaller $\alpha$ motor neurons or $\gamma$ motor neurons, throughout the spinal cord (Fig. 1, A to E, and fig. S2, A to F). Moreover, motor pools innervating predominantly fast or slow/ intermediate muscles respectively exhibited either high or low proportions of Dlk $1^{+}$motor neurons (Fig. 1, F to H, and fig. S2, C and D), together indicating selective expression of Dlk1 by fast motor neurons (fig. S2G).

To test whether Dlk1 would be involved in motor neuron functional diversification, we performed whole-cell patch clamp recordings of by cholera toxin B (CTB) (scale bar, $20 \mu \mathrm{m})$. late-gestation chick motor neurons engineered to stably express excess Dlk1 or control fluorescent protein (fig. S3). Excess Dlk1 shifted biophysical properties toward a profile typical of fast motor neurons (Fig. 2, A and B, and fig. S4, A to E), including elevated firing thresholds and frequencies and reduced afterhyperpolarization and firing periods (Fig. 2C and fig. S4, F to I).

We next analyzed motor neuron biophysical properties in acute spinal cord preparations of mice with the $D l k 1$ gene knocked out $\left(D l k 1^{K O}\right)$ (fig. S5A). Motor neurons in $D l k 1^{K O}$ mice showed a shift in biophysical properties opposite to those driven by excess Dlk1 in the chick (Fig. 2C and fig. S5, B to F). In normal mice, the proportion of $\mathrm{Dlk}^{+}$motor neurons $(34 \%)$ matched the proportion of motor neurons with a fast signature (30 to $32 \%$ ) (Fig. 2, D and E, and fig. S6, A and B). In $D l k l^{K O}$ mice, a similar proportion $(30 \%)$ of motor neurons shifted to lower firing thresholds (Fig. 2D and fig. S6, E to G) and slow/intermediate biophysical signatures, resulting in an almost complete lack of motor neurons with a fast signature (Fig. 2F and fig. S6, C and D). Together, these data indicated that Dlk1 is both sufficient and necessary for promoting a fast biophysical signature in motor neurons.

To test how a shift away from fast toward slow/ intermediate motor neuron properties would affect neuromuscular function, we analyzed the gait of mice selectively lacking Dlk1 in the motor neuron lineage (Dlk1 ${ }^{C K O}$ ) (fig. S7, A to D). Neither $D l k 1^{C K O}$ nor $D l k 1^{K O}$ mice showed measurable alterations in gait kinematics, posture, cutaneous sensation, rotarod test, or water maze performance (Fig. 3B and fig. S7, E to N). However, Dlk1 ${ }^{C K O}$ mice were deficient in braking and, to a lesser extent, propulsion velocities (Fig. 3A and fig. S7O), suggesting an inability to elicit the high forces needed in the extensor phase of the gait cycle (13). Consistently, Dlk1 ${ }^{C K O}$ mice showed abnormally low maximal limb force generation (Fig. 3C). Loss of a fast motor neuron biophysical signature in these mice thus resulted in deficient peak force outputs.
Fig. 1. Dlk1 is expressed by fast motor neurons. (A to C) Dlk1 expression in subsets of motor neurons colabeled by green fluorescent protein (GFP) in a postnatal day (P10) Hb9::eGFP transgenic mouse at brachial (A), thoracic (B), and lumbar (C) levels (scale bar, $150 \mu \mathrm{m}$ ). (D) Motor neuron (MN) size distribution in P10 mouse spinal cord: Dlk1 expression by the largest $\alpha$ motor neuron $\left(\mathrm{NeuN}^{+} \mathrm{Hb}^{+}\right)$size classes $[n=$ 450 motor neurons, three P10 mice; error bars indicate the standard error of the mean (SEM)]. (E) Dlk1 expression by a subset of $\mathrm{NeuN}^{+} \alpha$ motor neurons, but not NeuN" putative $\gamma$ motor neurons (open arrowhead) at P10. Scale bar, $10 \mu \mathrm{m}$. (F to $\mathbf{H})$ High abundance
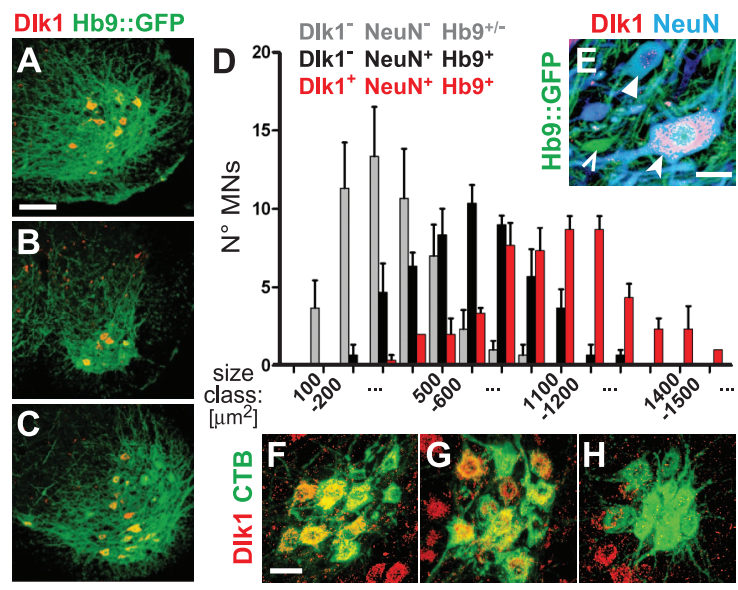
of Dlk1 $1^{+}$motor neurons in rectus femoris $(\mathrm{F})$, tibialis anterior $(\mathrm{G})$, but not soleus $(\mathrm{H})$ motor pools colabeled 
Through transcriptome profiling (fig. S8, A to C) we found that the shift of biophysical signatures in Dlk1-deficient motor neurons was accompanied by a shift in the expression of genes related to motor neuron type (Fig. 4A) but not of genes linked to generic or positional motor neuron identities (fig. S8, D and E), nor did we ob- serve altered abundance of $\gamma$ motor neurons (fig. S8, G to I). $58 \%$ of genes normally expressed by predominantly fast tibialis anterior and quadriceps motor pools were down-regulated, whereas genes normally expressed by the slow/intermediate soleus pool were up-regulated in the fast pools (Fig. 4A and fig. S8C).
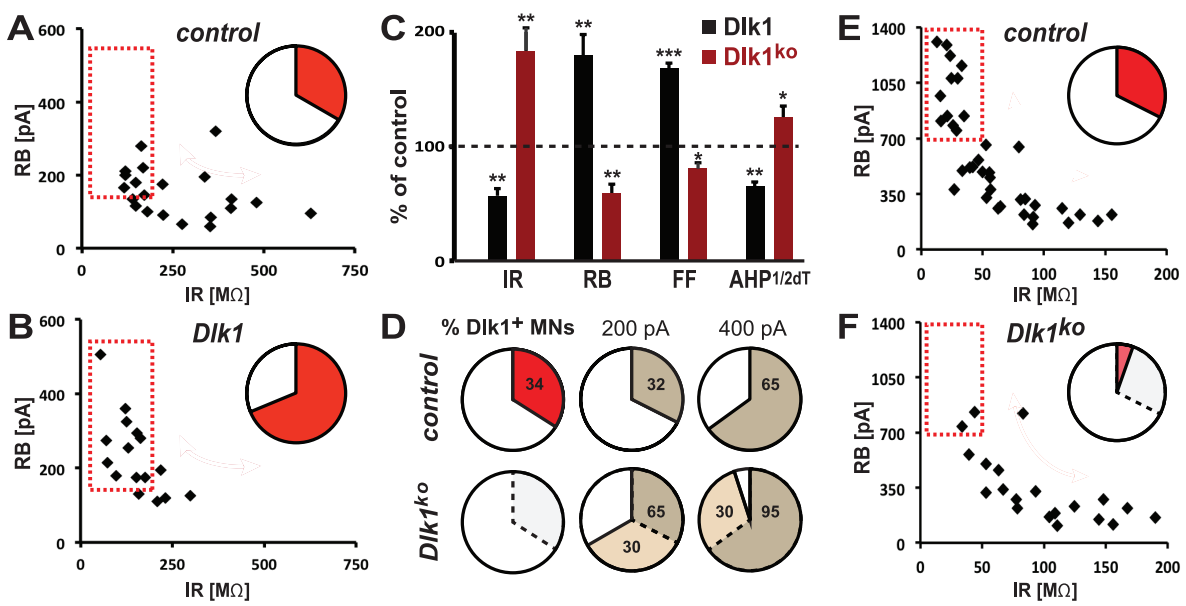

Fig. 2. Dlk1 is sufficient and necessary to promote a fast biophysical signature in motor neurons. (A and B) Biophysical signatures of control (GFP-transfected) (A) and Dlk1-transfected (B) embryonic day E12 to 15 chick motor neurons, based on rheobase (RB) against input resistance (IR) (4): Dlk1 promotes a shift toward a fast signature. Pie charts show the proportions of motor neurons inside the "fast quadrant" (arbitrarily delineated in red). (C) Black bars show that excess Dlk1 promotes a shift toward a fast biophysical signature: $R^{\text {high }}, \mathrm{IR}^{\text {low }}$, firing frequency (FF) ${ }^{\text {high }}$, and afterhyperpolarization half-decay time (AHPdT) ${ }^{\text {short }}(n=21$ control, 16 Dlk1 motor neurons). Red bars indicate a converse shift toward a slow biophysical signature in $D l k 1^{K O}\left(D l k 1^{-/-}\right)$as compared to control $\left(D l k 1^{+++}\right)$mice: $\mathrm{RB}^{\text {low }}, \mathrm{IR}^{\text {high }}, \mathrm{FF}^{\text {low }}$, $\mathrm{AHPdT}^{\text {long }}$ ( $n=37$ control, 21 Dlk1 ${ }^{K O}$ motor neurons; tables S3 to S5). (D) Percentage of Dlk1 $1^{+}$motor neurons in P10 mice (34 \pm 4\% SEM, 340 motor neurons, three P10 mice). A similar percentage (30\%) of $D l k 1^{K O}$ motor neurons were prematurely recruited to repetitive firing. (E to F) Biophysical signatures of control (E) and $D l k 1^{K O}$ motor neurons (F) (boxed quadrant, pie charts: subpopulation with a fast signature): loss of motor neurons with a fast signature in $D l k 1^{K O}$ mice (F). Paired two-tailed $t$-test in (C). $\left.{ }^{*} P<0.05\right)$, ${ }^{* * P} P$ $0.01,{ }^{* * *} P<0.001$. Error bars indicate SEM.
A Dlk1-dependent gene normally associated with a fast motor pool transcriptome signature was Keng4 (Fig. 4B and fig. S9, A to D), encoding a $\beta$ subunit of delayed-rectifier $\mathrm{K}^{+}$channels (14). Because these channels help tune neuronal firing properties (15) and are expressed by early postnatal mouse motor neurons (16), we asked whether Kcng4 could influence motor neuron properties. Similar to Dlk1, excess Kcng4 promoted elevation of rheobase and firing frequency, while shortening repetitive firing periods (Fig. 4C and fig. S9, E to H). However, unlike Dlk1, excess Keng4 did not shift other motor neuron properties (Fig. 4D

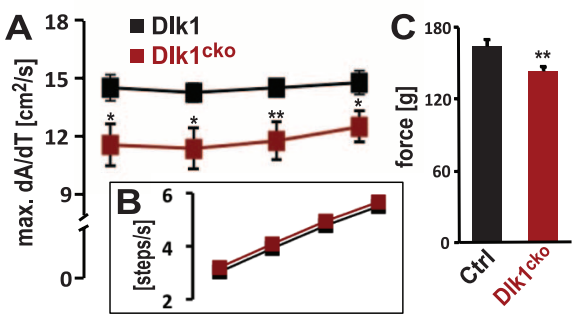

Fig. 3. Reduced peak force generation upon motor neuron-specific Dlk1 elimination. (A and $\mathbf{B})$ Gait analysis during brief running tasks at $10,20,30$, and $40 \mathrm{~cm} / \mathrm{s}$. (A) Reduced deceleration rates [maximal change of paw area $(d A)$ over time $(d T)$ ] during the extensor phase of the gait cycle in $D l k 1^{C K O}\left(D l k 1^{f x / f x} ;\right.$ Olig2 $\left.{ }^{\text {Cre }}\right)$ as compared to control $\left(D l k 1^{f \times / f x}\right)$ mice $\left(n=11,9\right.$ control $D l k 1^{C K O}$ mice, an average of three runs per condition). (B) Indistinguishable adaptation of stride frequency to increased running speeds by control and Dlk1 $1^{C K O}$ mice. (C) Reduced maximal limb force generation in $D l k 1^{\text {CKO }}$ mice ( $\mathrm{n}=11,9$ control $D l k 1^{\text {CKO }}$ mice). Paired two-tailed $t$ test was used in (A) to (C). ${ }^{*} P<$ $0.05,{ }^{* *} P<0.01$. Error bars indicate SEM.
Fig. 4. Dlk1 is required for motor neuron type-specific gene expression, including the neural activity modulator Kcng4. (A) Motor pool transcriptome signatures (heat maps) showing the loss of fast [quadriceps (QC) and tibialis anterior (TA)] signatures and a shift toward a slow/intermediate [soleus (SL)] signature in Dlk1 ${ }^{K O}$ mouse QC/TA pools ( $n=4$ mice per pool, cutoff $\geq 1.5$ fold, $P<0.05$ ). (B) Loss of differential Kcng4 expression between TA/SL pools in P4 Dlk1 ${ }^{K O}$ mouse. (C) Excess Kcng4 ( $n=16$ motor neurons) partially recapitulates the promotion of fast properties by Dlk1 ( $n=16$ motor neurons) in chick motor neurons. Noncleavable Dlk1 ${ }^{\mathrm{NC}}$ ( $n=10$ motor neurons), but not extracellular Dlk1 ${ }^{\mathrm{ES}}$ ( $n=14$ motor neurons) nor Dll1 ( $n=17$ motor neurons), recapitulates Dlk1 activity. Excess Dlk1 had no effect on adjacent nontransfected motor neurons (Dlk1 ${ }^{\mathrm{AD}]}, n=26$ motor neurons). Notch1 intracellular segment (NICD) abolishes Dlk1 effects on motor neuron properties ( $n=10$ motor neurons) (tables S6 and S7). (D) Dlk1 abolishes induction of the UAS::luciferase reporter by Notch1:Gal4 in Xenopus embryos. Luciferase normalized to constitutive Renilla fluorescence ( $n=3$ samples per condition in three experiments). Analysis of variance (ANOVA) was used in (A) and two-tailed $t$ test in (B) to (D). ${ }^{*} P<0.05,{ }^{* *} P<0.01,{ }^{* * *} P<0.001$. Error bars indicate SEM.

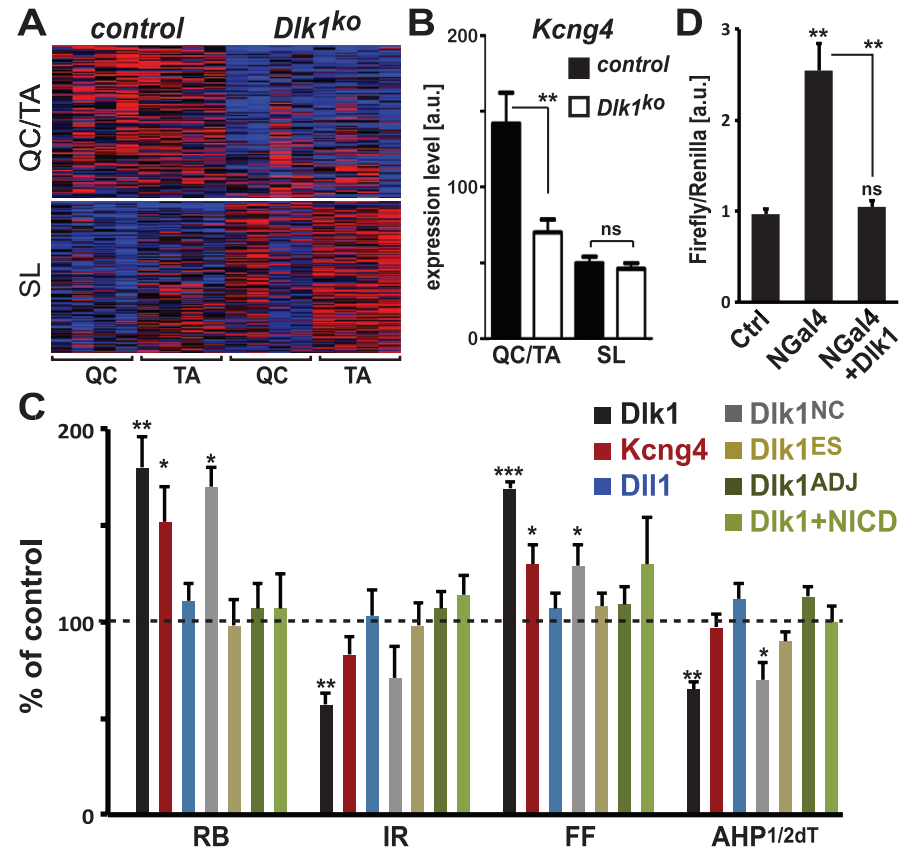


and fig. S9I). Thus, some but not all biophysical properties driven by Dlk1 are mediated by the secondary actor Keng4.

The Dlk1 isoforms expressed in the mouse spinal cord can give rise to membrane-tethered or cleaved extracellular proteins (fig. S10, A and B) $(11,12)$. We therefore forcedly expressed a noncleavable form of Dlk1 (Dlk1 $\left.1^{\mathrm{NC}}\right)$ or the extracellular segment of Dlk1 (Dlk1 ${ }^{\mathrm{ES}}$ ) (fig. S10B) in chick motor neurons. We observed that $\mathrm{Dlk} 1^{\mathrm{NC}}$, but not Dlk1 ${ }^{\mathrm{ES}}$, promoted fast properties (Fig. 4C). We further observed that only motor neurons forcedly expressing Dlk1, but not adjacent nontransfected motor neurons (fig. S10C), exhibited altered properties (Fig. 4C), together suggesting that Dlk1 operates cell-autonomously to promote a fast biophysical signature.

In preadipocytes, Dlk1 actions involve the inhibition of Notch signaling (17). Indeed, our expression of Dlk1 completely abolished the induction of a reporter for Notch activation in Xenopus embryos (Fig. 4D and fig. S10D). Moreover, forced expression of the canonical Notch activator Deltalike 1 (18) did not recapitulate the effects of excess Dlk1 on chick motor neuron properties (Fig. 4C). Furthermore, cotransfection of constitutively active Notch1 abolished the ability of excess Dlk1 to alter motor neuron properties (Fig. 4B), suggesting that Dlk1 action in motor neurons relies on Notch inhibition. Because Notch signaling is generally involved in cell fate decisions (18), it is likely that Dlk1 action involves additional pathways to promote fast motor neuron identity.

Here we have shown that Dlk1 is both necessary and sufficient for determining fast motor neurons and their corresponding biophysical signature in the mouse and chick (fig. S10E). Dlk1 implements expression of motor neuron type-specific genes such as Kcng4, which modulates a subset of neural activity parameters. The result is a biophysical signature in motor neurons that supports peak neuromuscular outputs. The strategy by which expression of a neural activity modulator is confined to a subset of neurons may similarly drive functional diversity elsewhere in the developing nervous system.

The overall lack of topographic organization for slow or fast motor neurons suggests that motor neuron type is acquired independently of the mechanisms that, before muscle innervation, determine motor neuron positional (column or pool) identities $(19,20)$. We still do not know when subsets of motor neurons acquire typespecific biophysical signatures, to what extent motor neuron functional diversification involves signals from muscle (21), how motor neuron and muscle fiber types are matched (22-24), or what causes the differential vulnerability of motor neuron types to disease or aging (25). However, 57 years after the characterization of fast and slow motor neurons (1), we can now have insight into the molecular mechanisms that control their development and function.

\section{References and Notes}

1. J. C. Eccles, R. M. Eccles, A. Lundberg, Nature 179, 866-868 (1957)

2. R. E. Burke, D. N. Levine, F. E. Zajac 3rd, P. Tsairis, W. K. Engel, Science 174, 709-712 (1971).

3. D. Kernell, The Motoneuron and Its Muscle Fibers (Oxford Univ. Press, New York, ed. 1, 2006).

4. J. E. Zengel, S. A. Reid, G. W. Sypert, J. B. Munson, 1. Neurophysiol. 53, 1323-1344 (1985).

5. R. Bakels, D. Kernell, J. Physiol. 463, 307-324 (1993).

6. M. Manuel, C. J. Heckman, J. Neurosci. $\mathbf{3 1}$ 15188-15194 (2011).

7. E. Henneman, Science 126, 1345-1347 (1957).

8. D. Kernell, Science 152, 1637-1639 (1966).

9. M. Gustafsson, M. J. Pinter, Trends Neurosci. 8, 431-433 (1985).

10. E. Davis et al., Curr. Biol. 14, 1858-1862 (2004).

11. H. S. Sul, Mol. Endocrinol. 23, 1717-1725 (2009).

12. S. R. Ferrón et al., Nature 475, 381-385 (2011).

13. S. Grillner, in Handbook of Physiology: The Nervous System, Motor Control, Vol. 2, V. B. Brooks, Ed. (American Physiological Society, Bethesda, MD 1981), pp. 1179-1236.

14. N. Ottschytsch, A. Raes, D. Van Hoorick, D. J. Snyders, Proc. Natl. Acad. Sci. U.S.A. 99, 7986-7991 (2002).

15. H. Murakoshi, J. S. Trimmer, J. Neurosci. 19, 1728-1735 (1999)
16. J. M. Wilson, ]. Rempel, R. M. Brownstone, J. Comp. Neurol. 474, 13-23 (2004).

17. M. L. Nueda, V. Baladrón, B. Sánchez-Solana, M. A. Ballesteros, ]. Laborda, J. Mol. Biol. 367 1281-1293 (2007).

18. K. Hori, A. Sen, S. Artavanis-Tsakonas, J. Cell Sci. 126 2135-2140 (2013).

19. W. A. Alaynick, T. M. Jessell, S. L. Pfaff, Cell 146, 178, e1 (2011).

20. ]. S. Dasen, T. M. Jessell, Curr. Top. Dev. Biol. 88 169-200 (2009).

21. ]. V. Chakkalakal, H. Nishimune, ]. L. Ruas, B. M. Spiegelman, ]. R. Sanes, Development 137, 3489-3499 (2010).

22. W. J. Thompson, L. A. Sutton, D. A. Riley, Nature 309 709-711 (1984).

23. T. Fladby, ]. K. Jansen, Development 109, 723-732 (1990).

24. V. F. Rafuse, L. D. Milner, L. T. Landmesser, J. Neurosci. 16, 6864-6877 (1996)

25. K. C. Kanning, A. Kaplan, C. E. Henderson, Annu. Rev. Neurosci. 33, 409-440 (2010).

Acknowledgments: We thank A. Klusowski, B. Veith, T. V. Bui and T. G. Hampton for technical support; S. Schiaffino and K. Kawakami for reagents; P. D'Adamo for phenotyping service; and $\mathrm{O}$. Shomroni and S. Bonn for bioinformatics advice. This research received funding from the European Research Council under the European Union's Seventh Framework Programme (FP/2007-2013)/ERC Grant Agreement 311710-MU TUNING and the Emmy-Noether Program of the Deutsche Forschungsgemeinschaft (MA4278/1-1). S.R.B. was supported by the Center for Biologics Evaluation and Research, Food and Drug Administration, operating funds; O.S. by the Emmy-Noether Program (SCHL592-4); R.M.B. by the Canadian Institutes of Health Research, Canada Research Chairs Program, Canada Foundation for Innovation, and Nova Scotia Research and Innovation Trust; and J.L. by the Ministerio de Educación y Ciencia, Spain, from whom $D l k 1^{k o}$ mice are available through a materials transfer agreement. $D l k 1^{f x}$ mice are deposited at jaxmice.jax.org (no. 019074). Notch1:Gal4 is available from R. Kopan (Cincinnati Children's Hospital Medical Center). ENI-G is a cooperation of University Medical Center Göttingen and the Max-Planck Society.

Supplementary Materials

www.sciencemag.org/content/343/6176/1264/suppl/DC1 Materials and Methods

Supplementary Text

Figs. S1 to S10

Tables $\$ 1$ to $\$ 9$

References (26-43)

25 September 2013; accepted 14 February 2014 $10.1126 /$ science. 1246448 

for Peak Force Execution

Daniel Müller et al.

Science 343, 1264 (2014);

DOI: $10.1126 /$ science. 1246448

This copy is for your personal, non-commercial use only.

If you wish to distribute this article to others, you can order high-quality copies for your colleagues, clients, or customers by clicking here.

Permission to republish or repurpose articles or portions of articles can be obtained by following the guidelines here.

The following resources related to this article are available online at www.sciencemag.org (this information is current as of April 7, 2015 ):

Updated information and services, including high-resolution figures, can be found in the online version of this article at:

http://www.sciencemag.org/content/343/6176/1264.full.html

Supporting Online Material can be found at:

http://www.sciencemag.org/content/suppl/2014/03/12/343.6176.1264.DC1.html

This article cites $\mathbf{4 1}$ articles, 20 of which can be accessed free:

http://www.sciencemag.org/content/343/6176/1264.full.html\#ref-list-1

This article appears in the following subject collections:

Neuroscience

http://www.sciencemag.org/cgi/collection/neuroscience 